\title{
RESISTÊNCIA MECÂNICA DO SOLO À PENETRAÇÃO NA CULTURA DO AMENDOIM EM DIFERENTES SISTEMAS DE MANEJO DO SOLO
}

\author{
Venâncio Betiol $^{(1,5)}$, Larissa Morais da Silva Ambrosio ${ }^{(1,4)}$, Rhanyel Tritula Barini ${ }^{(1,5)}$, Leonardo \\ Henrique de Oliveira Gonçalves ${ }^{(1)}$, Élcio Rios Pérez Leal ${ }^{(1)}$, Denizart Bolonhezi ${ }^{(1,3)}$
}

${ }^{1}$ Graduandos em Agronomia, Instituto Moura Lacerda, Ribeirão Preto, SP, Email: venanciobetiol@hotmail.com (Apresentador do trabalho), ${ }^{2}$ Orientador e Pesquisador Científico da APTA, Ribeirão Preto, SP, Email: dbolonhezi@gmail.com, ${ }^{3}$ Bolsista em Desenvolvimento Tecnológico do CNPQ, ${ }^{4}$ Bolsista de Iniciação Científica do CNPQ, ${ }^{5}$ Bolsista de Iniciação Científica da FUNDAG.

RESUMO: Pesquisa de campo foi conduzida em Assis/SP, em condição de Latossolo Vermelho, textura arenosa, com objetivo de avaliar a objetivo avaliar a variação em profundidade da resistência mecânica do solo à penetração em diferentes posições de leitura (linha e entrelinha), épocas de avaliação e sistemas de preparo de solo para a cultura do amendoim cultivado em reforma de canavial. Houve interação significativa entre o fator sistemas de manejo com os outros fatores (profundidade e posição de leitura), para medidas efetuadas em janeiro e fevereiro. Todavia, em março verificou-se diferença estatística da RMSP somente entre camadas de amostragem. Não houve diferença estatística entre os manejos nas camadas superficiais, enquanto abaixo de $40 \mathrm{~cm}$ foram verificados os maiores valores de RMSP, sobretudo para os manejos conservacionistas.

Palavras-Chave: Arachis hypogaea L., plantio direto, preparo reduzido, compactação do solo.

\section{INTRODUÇÃO}

Atualmente, mais de $80 \%$ dos canaviais paulistas (5.76 milhões de ha) são colhidos sem queima prévia (cana crua), e a partir de 2017 mesmo nas áreas com declividade acima de 12\% o fogo não será permitido no Estado de São Paulo. Nestas condições, é desejável a adoção de sistemas conservacionistas de manejo do solo na reforma do canavial, pois esta reduz em $30 \%$ os custos com preparo e reduz em 10 vezes a erosão (BOLONHEZI et al., 2014). Contudo, os produtores de amendoim ainda não se adaptaram a esta mudança, fato que dificulta o estabelecimento de parcerias de arrendamento e consequentemente migração em busca de terras para cultivo nos Estados vizinhos, refletindo em aumento no custo de produção e comprometimento da cadeia produtiva paulista.

Embora já exista um mínimo de lastro técnico-científico gerado sobre a viabilidade do amendoim em manejo conservacionista, algumas questões, tais como; perdas na colheita, impacto da compactação do solo sobre o desenvolvimento das plantas, estabelecimento do estande inicial e níveis de aflatoxina, ainda necessitam ser validadas em escala comercial. As pesquisas realizadas na última década para as condições norte-americanas, tem utilizado com frequência um equipamento denominado de Rip Strip ${ }^{\circledR}$ da KMC (Kelley Manufacturing Co.). Este equipamento realiza preparo em faixas entre 20 e $46 \mathrm{~cm}$ de largura através de quatro discos corrugados posicionados na vertical e entre 
25 e $45 \mathrm{~cm}$ de profundidade, através de uma haste subsoladora. Siri-Prieto et al. (2009) estudaram o uso deste implemento em comparação com a semeadura direta, em integração com pecuária. Concluíram que o maior lucro foi obtido com o preparo em faixa (US\$ 462), em comparação à semeadura direta sobre pastagem (US\$ 41). Em vista do exposto, o trabalho teve como objetivo avaliar a variação em profundidade da resistência mecânica do solo à penetração em diferentes posições de leitura (linha e entrelinha), épocas de avaliação e sistemas de preparo de solo para a cultura do amendoim cultivado em reforma de canavial.

\section{MATERIAL E MÉTODOS}

$\mathrm{O}$ ensaio foi instalado em Assis/SP, em áreas de canavial com histórico de 7 cortes mecanizados e solo classificado como LATOSSOLO Vermelho, com textura arenosa e a quantidade de palha quantificada antes da instalação foi de aproximadamente $10,9 \mathrm{Mg} \mathrm{ha}^{-1}$ de matéria seca de palhiço. Como práticas corretivas, foram aplicados $1,7 \mathrm{Mg} \mathrm{ha}^{-1}$ de calcário, $1.0 \mathrm{Mg} \mathrm{ha}^{-1}$ de gesso agrícola e $350 \mathrm{~kg} \mathrm{ha}^{-1}$ de fosfato. Após aplicação dos corretivos, as parcelas com dimensões de 14,4 x $30 \mathrm{~m}$ foram demarcadas, seguindo delineamento experimental blocos ao acaso com 7 repetições e espaçamento entre linhas de $0,90 \mathrm{~m}$. O tratamento convencional consistiu da passagem de grade intermediária, seguida por gradagem aradora, novamente uma gradagem intermediária e finalizado com gradagem niveladora. $\mathrm{O}$ tratamento denominado preparo reduzido foi realizado com equipamento Rip Strip ${ }^{\circledR}$ da KBM Dumont, com 6 hastes, razão pela qual houve necessidade de remontar algumas linhas, considerando que a semeadura foi efetuada com semeadora da marca Jumil modelo 2680PD de quatro linhas e com disco corta palha para plantio direto. A variedade utilizada de amendoim foi a IAC-OL3. Para realizar as amostragens das avaliações de resistência mecânica do solo à penetração (RMSP), foi utilizado um carrinho de duas rodas acoplado ao mesmo um penetrógrafo eletrônico digital, marca (DLG PNT 2000), o qual penetra o solo com uma haste com força constante, a uma profundidade de até $0,54 \mathrm{~m}$ e as leituras foram obtidas através de um dinamômetro digital. Os dados foram obtidos através de 5 leituras por parcela nas entrelinhas e 5 nas linhas de semeadura do amendoim, nos três sistemas de manejo do solo. As coletas dos dados foram feitas nos dias 06/01/2017, 08/02/2017 e 18/03/2017. Nesse mesmo dia, foram retiradas 1 amostra de solo simples por parcela, em diferentes camadas de solo $(0-10,10-20,20-30,30-40$ e 40-50 cm), para determinação da umidade pelo método gravimétrico (secagem a temperatura de $\pm 100{ }^{\circ} \mathrm{C}$ em estufa com circulação forçada de ar quente). Os dados foram submetidos a análise da variância usando software estatístico AgroEstat (FCAVJ), considerando a profundidade e posição de leitura como fatores em um delineamento em parcelas sub-subdivididas. As médias foram comparadas de acordo com teste de Tukey $(5 \%)$.

\section{RESULTADOS E DISCUSSÃO}

Verifica-se na Tabela 1, que houve interação significativa entre os fatores manejo x camada do dolo, manejo do solo x posição da leitura e camada do solo x posição da leitura e no mês de fevereiro, 
a interação tripla manejo $\mathrm{x}$ camada de solo $\mathrm{x}$ posição de leitura foi significativa, portanto a interpretação dos resultados deve ser feita através dos desdobramentos dessas interações. Por outro lado, no mês de março não foi verificada dependência entre os fatores estudados, nesse caso verificouse diferença estatística somente entre as camadas do solo consideradas na análise. É interessante notar que após 120 dias (abril) da realização dos preparos, somente foi identificada diferença estatística entre as camadas de solo consideradas, não sendo mais observados os contrastes entre os tratamentos de preparo de solo.

Tabela 1. Resistência mecânica do solo à penetração (MPa) em diferentes manejos de solo, camada do solo e posição de leitura, avaliados em três épocas diferentes, na cultura do amendoim IAC-OL3, cultivado na reforma de cana crua. Assis, SP, 2017.

\begin{tabular}{|c|c|c|c|}
\hline Manejos de Solo (M) & Janeiro & Fevereiro & Março \\
\hline Preparo Convencional & $1.24 \mathrm{~b}$ & $1.89 \mathrm{~b}$ & $2.05 \mathrm{a}$ \\
\hline Rip Strip & $1.91 \mathrm{a}$ & $2.50 \mathrm{a}$ & $2.04 \mathrm{a}$ \\
\hline Plantio Direto & $2.10 \mathrm{a}$ & $2.64 \mathrm{a}$ & $2.44 \mathrm{a}$ \\
\hline Teste $\mathrm{F}$ & $9.6 * *$ & $23.0 * *$ & $2.69 \mathrm{~ns}$ \\
\hline d.m.s. (Tukey 5\%) & 0.55 & 0.31 & 0.52 \\
\hline \multicolumn{4}{|l|}{ Camada de Solo (C) } \\
\hline $0-10$ & $0.25 \mathrm{e}$ & $0.41 \mathrm{~d}$ & $0.33 \mathrm{~d}$ \\
\hline $11-20$ & $0.99 \mathrm{~d}$ & $1.72 \mathrm{c}$ & $1.50 \mathrm{c}$ \\
\hline $21-30$ & $1.89 \mathrm{c}$ & $2.74 \mathrm{~b}$ & $2.56 \mathrm{~b}$ \\
\hline $31-40$ & $2.67 \mathrm{~b}$ & $3.38 \mathrm{a}$ & $3.12 \mathrm{a}$ \\
\hline $41-54$ & $2.95 \mathrm{a}$ & $3.43 \mathrm{a}$ & $3.39 \mathrm{a}$ \\
\hline Teste F & $310.6 * *$ & $994.2 * *$ & $254,0 * *$ \\
\hline d.m.s. (Tukey 5\%) & 0.25 & 0.16 & 0.31 \\
\hline \multicolumn{4}{|l|}{ Posição de Leitura (P) } \\
\hline Linha & $1.56 \mathrm{~b}$ & $2.25 \mathrm{~b}$ & $2.13 \mathrm{a}$ \\
\hline Entrelinha & $1.94 \mathrm{a}$ & $2.42 \mathrm{a}$ & $2.23 \mathrm{a}$ \\
\hline Teste F & $55.3 * *$ & $10.3 * *$ & $1.23 \mathrm{~ns}$ \\
\hline d.m.s. (Tukey 5\%) & 0.10 & 0.10 & 0.19 \\
\hline \multicolumn{4}{|l|}{ Interações } \\
\hline $\mathrm{M} \times \mathrm{C}$ & $5.96 * *$ & $24.89 * *$ & $1.13 \mathrm{~ns}$ \\
\hline $\mathrm{M} \times \mathrm{P}$ & $6.92 * *$ & $4.94 * *$ & $0.98 \mathrm{~ns}$ \\
\hline $\mathrm{C} \times \mathrm{P}$ & $14.3 * *$ & $17.16 * *$ & $0.96 \mathrm{~ns}$ \\
\hline $\mathrm{M} \times \mathrm{C} \times \mathrm{P}$ & $0.97 \mathrm{~ns}$ & $2.5 *$ & $0.44 \mathrm{~ns}$ \\
\hline C.V. (\%) manejos & 69,9 & 29,8 & 51,4 \\
\hline C.V. $(\%)$ camadas & 23,8 & 11,2 & 23,6 \\
\hline C.V. (\%) posições & 21,0 & 16,2 & 31,9 \\
\hline
\end{tabular}

Médias seguidas de mesma letra minúscula na coluna não diferem entre si pelo teste de Tukey.

$\mathrm{Na}$ Tabela 2 pode-se observar que até $20 \mathrm{~cm}$ de profundidade não foi identificada diferença estatística entre os tratamentos de preparo do solo. Todavia, houve diferença altamente significativa entre os tratamentos nas demais camadas, com valores máximos $(3,26 \mathrm{MPa})$ verificados entre 41 e 54 $\mathrm{cm}$ de profundidade nos manejos conservacionistas plantio direto e Rip Strip. O máximo valor de RMSP encontrado nos dois sistemas de manejo conservacionistas, considerando a média entre linha e entrelinha pode ser considerados como médio (> 2 a 4 MPa) segundo Roque et al. (2003). Contudo, esse valor ultrapassa os níveis considerados aceitáveis para o pleno desenvolvimento do sistema radicular, que segundo Canarache (1990) situam-se no limite máximo de 2,5 MPa.

Considerando a média entre as camadas de solo, foram verificadas diferenças estatísticas entre a posição de leitura somente para os manejos conservacionistas (Tabela 3). Na linha de semeadura a RMSP foi significativamente maior no plantio direto, seguido pelo Rip Strip que não diferiu do convencional, demonstrando um benefício do preparo localizado (Tabela 4). Entretanto, para a média 
dos preparos, nenhuma diferença estatística foi verificada entre linha e entrelinha abaixo dos $31 \mathrm{~cm}$ de profundidade. Bolonhezi et al. (2016) testaram esses mesmos tratamentos e encontraram maiores valores da RMSP abaixo dos $30 \mathrm{~cm}$ no sistema convencional, nas medições efetuadas após as operações de colheita, porém sem discriminar as leituras na linha e entrelinha.

Tabela 2. Desdobramento da interação dos fatores Manejo x Camada do Solo, para a resistência mecânica do solo à penetração (MPa), avaliados no mês de janeiro, na cultura do amendoim IAC-OL3, cultivado na reforma de cana crua. Assis, SP, 2017.

\begin{tabular}{ccccccc}
\hline & $0-10$ & $11-20$ & $21-30$ & $31-40$ & $41-54$ & Teste $\mathrm{F}$ \\
\hline Preparo Convencional & $0.11 \mathrm{Da}$ & $0.67 \mathrm{Ca}$ & $1.30 \mathrm{Bb}$ & $1.78 \mathrm{Ab}$ & $2.33 \mathrm{Ab}$ & $62.11^{* *}$ \\
Rip Strip & $0.31 \mathrm{Da}$ & $1.07 \mathrm{Ca}$ & $1.94 \mathrm{Ba}$ & $2.98 \mathrm{Aa}$ & $3.26 \mathrm{Aa}$ & $126.4^{* *}$ \\
Plantio Direto & $0.32 \mathrm{Ea}$ & $1.25 \mathrm{Da}$ & $2.44 \mathrm{Ca}$ & $3.23 \mathrm{Ba}$ & $3.26 \mathrm{Aa}$ & $134.02^{* *}$ \\
\hline Teste F & $0.40 \mathrm{~ns}$ & $2.84 \mathrm{~ns}$ & $10.6^{* *}$ & $19.07 * *$ & $9.16^{* *}$ & \\
\hline
\end{tabular}

Médias seguidas de mesma letra minúscula na coluna e maiúscula na linha, não diferem entre si pelo teste de Tukey $(5 \%)$

Tabela 3. Desdobramento da interação dos fatores Manejo x Posição de Leitura, para a resistência mecânica do solo à penetração $(\mathrm{MPa})$, avaliados no mês de janeiro, na cultura do amendoim IAC-OL3, cultivado na reforma de cana crua. Assis, SP, 2017.

\begin{tabular}{cccc}
\hline & Linha & Entrelinha & Teste F \\
\hline Preparo Convencional & $1.18 \mathrm{Ab}$ & $1.30 \mathrm{Ab}$ & $1.89 \mathrm{~ns}$ \\
Rip Strip & $1.69 \mathrm{Bab}$ & $2.13 \mathrm{Aa}$ & $25.27 * *$ \\
Plantio Direto & $1.82 \mathrm{Ba}$ & $2.38 \mathrm{Aa}$ & $42.00^{* *}$ \\
\hline Teste F & $4.86^{*}$ & $13.81^{* *}$ & \\
\hline
\end{tabular}

Médias seguidas de mesma letra minúscula na coluna e maiúscula na linha, não diferem entre si pelo teste de Tukey $(5 \%)$

Tabela 4. Desdobramento da interação dos fatores Posição de Leitura x Camada do Solo, para a resistência mecânica do solo à penetração (MPa), avaliados no mês de janeiro, na cultura do amendoim IAC-OL3, cultivado na reforma de cana crua. Assis, SP, 2017.

\begin{tabular}{ccccccc}
\hline & $0-10$ & $11-20$ & $21-30$ & $31-40$ & $41-54$ & Teste $\mathrm{F}$ \\
\hline Linha & $0.11 \mathrm{Db}$ & $0.50 \mathrm{Cb}$ & $1.61 \mathrm{Bb}$ & $2.69 \mathrm{Aa}$ & $2.94 \mathrm{Aa}$ & $221.03^{* *}$ \\
Entrelinha & $0.42 \mathrm{Ca}$ & $1.51 \mathrm{Ca}$ & $2.18 \mathrm{Ba}$ & $2.64 \mathrm{Aa}$ & $2.96 \mathrm{Aa}$ & $140.02 * *$ \\
\hline Teste $\mathrm{F}$ & $8.63^{* *}$ & $78.14^{* *}$ & $25.36^{* *}$ & $0.15 \mathrm{~ns}$ & $0.04 \mathrm{~ns}$ & \\
\hline
\end{tabular}

Médias seguidas de mesma letra minúscula na coluna e maiúscula na linha, não diferem entre si pelo teste de Tukey $(5 \%)$

Os resultados referentes ao mês de fevereiro encontram-se nos gráficos da Figura 1, bem como a variação da RMSP em cada tratamento relativa ao mês de março. Considerando a média entre as duas posições de leitura, somente na camada de $0-10 \mathrm{~cm}$ não foi identificada diferença estatística entre os tratamentos de preparo de solo. Para a média das posições de leitura, vale notar que abaixo de 41 $\mathrm{cm}$, as maiores RMSP foram verificadas no tratamento Rip Strip (3,93 MPa), seguido pelo plantio direto $(3,50 \mathrm{MPa})$ e preparo convencional $(2,85 \mathrm{MPa})$, todas estatisticamente diferentes (Tukey5\%). Provavelmente, a ponteira da haste subsoladora do equipamento Rip Strip ocasionou um "espelhamento" devido a alta umidade do solo nas camadas subsuperficiais, por ocasião da instalação do tratamento em dezembro de 2016. Consequentemente, os valores de RMSP medidos foram significativamente maiores que no tratamento convencional e plantio direto, mesmo com maior conteúdo de água no solo nessa camada no Rip Strip (Figura 2). 


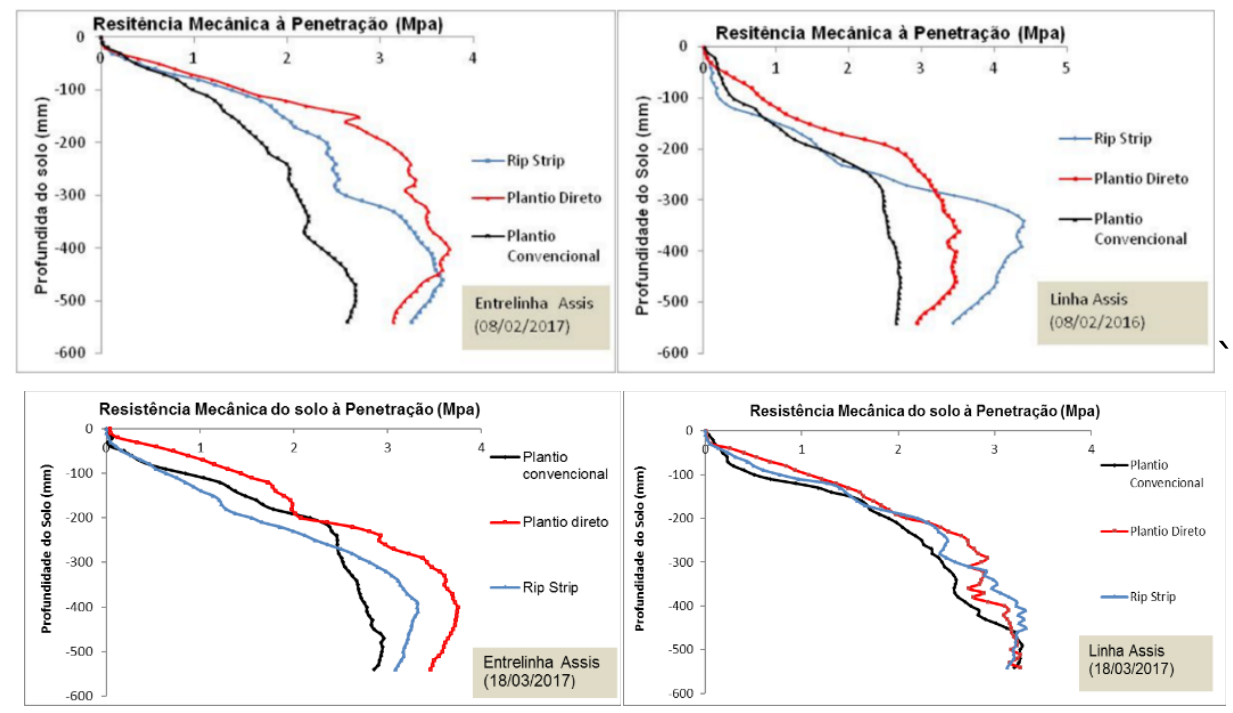

Figura 1. Resistência à penetração (MPa) medida na linha e entrelinha em diferentes sistemas de manejo de solo na reforma de canavial na região de Assis/SP. Média de 35 repetições.

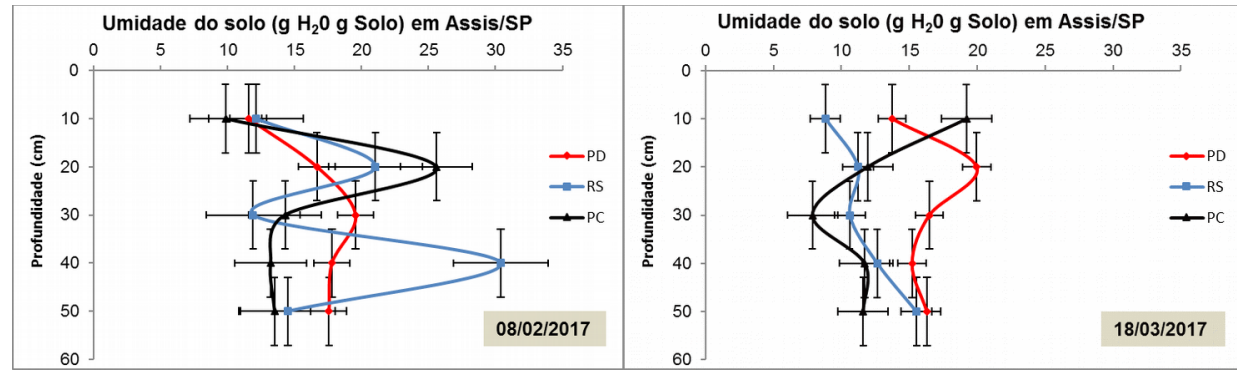

Figura 2. Umidade do solo do ensaio da Região de Assis/SP em diferentes datas de amostragens e profundidades.

\section{CONCLUSÕES}

A resistência mecânica do solo à penetração nos diferentes sistemas de manejo dependeu da profundidade de amostragem e da posição de avaliação, para as medidas efetuadas em janeiro e fevereiro, mas sem efeito quando realizadas em março. Até $20 \mathrm{~cm}$ não foram verificadas diferenças significativas entre os sistemas de manejo, contudo abaixo de $40 \mathrm{~cm}$, os maiores valores foram obtidos no tratamento Rip Strip (3,93 MPa), seguido pelo plantio direto (3,50 $\mathrm{MPa})$ e preparo convencional $(2,85 \mathrm{MPa})$.

\section{AGRADECIMENTOS}

Ao CNPQ pelas bolsas de iniciação científica PIBIT e de produtividade em desenvolvimento tecnológico DT-2. À KBM Dumont pelo empréstimo do equipamento Rip Strip, e a Usina Estiva, por todo apoio na condução desta pesquisa. À DLG Automação pelo fornecimento do penetrógrafo digital PNT2000.

\section{REFERENCIAS BIBLIOGRÁFICAS}

BOLONHEZI, D.; ROSSINI, D.B.; FRIZZAS, A.; FURLANI, C.E. A. de; BOLONHEZI, A.C. Conservation agriculture principles applied for brazilian peanut crop system. In: WORLD CONGRESS ON CONSERVATION AGRICUlTURE, VI. Proceedings..., Winnipeg, Canadá, p.140-144, 2014. CD-Rom. 
BOLONHEZI, D.; VALOCHI, R.; ZANANDRÉA, P.C.; CARVALHO, E.V.; CARDOSO, B.M.V.; RAMOS, G.; BETIOL, M.B.; SCARPELLINI, J.R.; BOLONHEZI, A.C. Perdas no arranquio de amendoim e resistência à penetração do solo em manejo conservacionista na reforma de canavial. In: WORKSHOP AGROENERGIA MATÉRIAS-PRIMAS, X. Anais... Ribeirão Preto, IAC. CD-Rom.

CANARACHE, A. PENETR - a generalized semi-empirical model estimating soil resistance to penetration. Soil Tillage Research, Amsterdam, v.16, n.1, p.51-70, 1990.

ROQUE, C. G.; CENTURION, J. F.; ALENCAR, G. V.; BEUTlER, A. N.; PEREIRA, G. T.; ANDRIOLI, I. Comparação de dois penetrômetros na avaliação de resistência à penetração de um latossolo vermelho sob diferentes usos. Acta Scientiarum (UEM), Maringá, v. 25, p. 53-57, 2003.

SIRI-PRIETO, G.; REEVES, D.W.; RAPER, R.L. Tillage requirements for integrating winter-annuel grazing in peanut production: plant water status and productivity. Agronomy Journal, 101(6):14001408, 2009. 